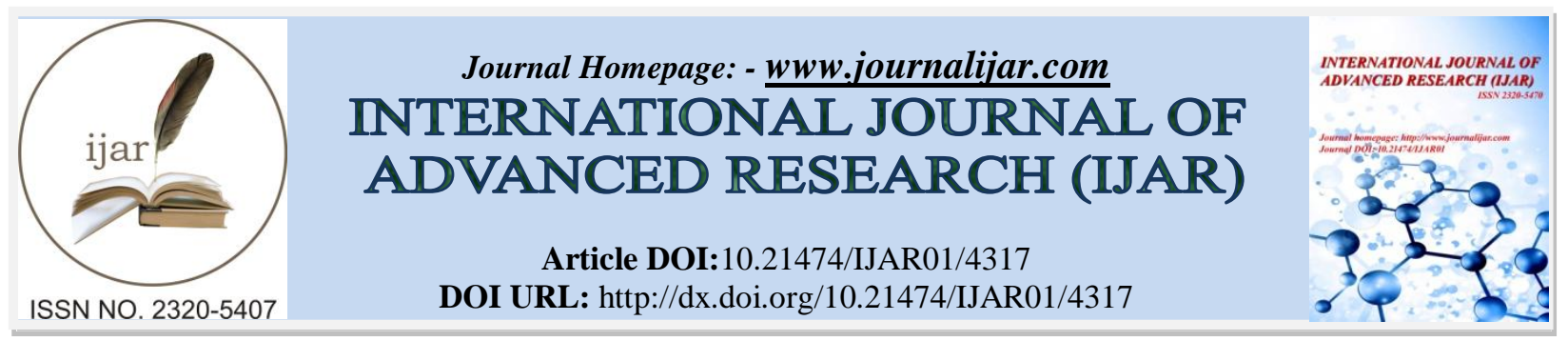

RESEARCH ARTICLE

\title{
PREVALENCE OF GROUP B STREPTOCOCCI COLONIZATION IN GEORGIAN PREGNANT WOMEN: COMMUNITY BASED CROSS SECTIONAL STUDY.
}

\author{
Tamar Didbaridze $^{1}$,Tinatin Gagua ${ }^{2}$, Nino Gogokhia ${ }^{3}$, David Gagua ${ }^{4}$, Nato Metskhvarishvili ${ }^{5}$, Maia \\ Rizhvadze $^{6}$ and Eka Shvelashvili ${ }^{7}$. \\ 1. TSMU The First University Clinic, department of Microbiology,. MD. PhD. \\ 2. Davit Tvildiani Medical University, MD, PhD. Department of obstetrics gynecology. \\ 3. Head of Clinical Laboratory. Professor. MD. PhD. TSMU the First University Clinic. \\ 4. Davit Tvildiani Medical Univeristy, MD, PhD. Head of Department of obstetrics gynecology. \\ 5. TSMU Department of Obstetrics and Gynecology, Assistant professor, MD, $\mathrm{PhD}$ (Tbilisi, Georgia). \\ 6. TSMU Department of Obstetrics and Gynecology, MD, PhD,(Tbilisi, Georgia). \\ 7. TSMU Department of Obstetrics and Gynecology, MD, PhD,(Tbilisi, Georgia).
}

\section{Manuscript Info}

Manuscript History

Received: 21 March 2017

Final Accepted: 27 April 2017

Published: May 2017

\section{Abstract}

Background: Group B streptococcus colonization in pregnant women usually asymptomatic, but it is one of the major factors of newborn infection in developed countries. In Georgia, there is no information about the prevalence of maternal colonization and newborns infected by group. We conducted a study of its prevalence among Georgian pregnant women.

Materials and Methods: This is a cross-sectional descriptive and analytic study performed at the prevalence of GBS colonization in pregnant women in two major university clinics: TSMU The First University and " Davit Tvildiani Medical University", Department of obstetrics gynecology in 2015-2016 in Tbilisi. The vaginal culture for group B streptococcus was done for 1648 mothers based on our protocol. Data was analyzed (SPSS version 15).

Results: Among 1648 pregnant women (aged 18-42 years) 362 had positive recto- vaginal GBS $(21,9 \%)$. No significant difference was found in positive cultures with mother's age, educational level, and history of pregnancy

Conclusion: The colonization of group B streptococcus in Georgian women is significant, therefore, screening of newborns for group B streptococcus infection is recommended to become a routine practice in all healthcare centers in Georgia.

Copy Right, IJAR, 2017,. All rights reserved.

\section{Introduction:-}

Recto-vaginal colonization of Group B streptococcus (GBS) is life threatening issue of newborn's bacterial infection and general health, which is getting frequent in developing countries. In 15-40\% Healthy women's colon and vagina is colonized by Group B streptococci (GBS). Pregnant women with colonized vagina with GBS frequently transmit it to their newborn [1],[3].In the recent decade, Group B Streptococcus (GBS) has been one of 
the common causes of the early onset of sepsis among the newborns, which leads to high rate of morbidity and mortality [1]. The incidence of early onset GBS disease is from 1.3 to 3.7 per 10000 live births [2]. GBS is also considered to be the leading cause of puerperal and intranatal infection: chorioamnionitis, endometritis, surgical wound infection and genitourinary tract. One-third of preterm deliveries is also associated with genital infection, GBS produce protease activity resulting to cervical ripening [3].

Even though the organism can be found in throat, vagina and rectum, vast majority of infected women remain asymptomatic [4] Age, race, concurrent vaginal yeast colonization, parity, genetic-ethnic factors, socio-economical status, consumption of pork and recent sexual intercourse are factors that influence the prevalence of colonization[4][5].

Early onset neonatal sepsis is a a result of vertical transmission from clonized mother before or during labor, [6]. The rate of vertical transmission of GBS between mothers and their offspring is about $29-85 \%$ (mean=51\%). This transmission depends on the severity of maternal colonization in birth canal [4].

Receiving antibiotic during delivery dramatically changes the outcome, reducing the GBS infection rate from one out of 200 to one out of 4000. The infection rate increases when other predisposing factors like premature rupture of membranes (PROM) more than 18 hours, prematurity, maternal fever, low birth weight and multi parity are present. [4]. The two major prevention strategies for GBS disease are: the screening method and the risk-based approach. Intrapartum antibiotic prophylaxis is offered to all pregnant women carrying GBS [7].

The Centers for Diseases Control (CDC) recommended GBS screening for all pregnant women between 35 and 37 weeks of pregnancy, as well as taking intrapartum antibiotic prophylaxis [8][9]. Pregnant women with unknown GBS status should be treated with antibiotic at the time of delivery [4]

The mortality rate of early onset sepsis has estimated about 50\% [9][10]. Furthermore, early onset GBS sepsis is also associated with serious neurological damage.

In our country, there is not information about maternal colonization and newborn infection with GBS. So We conducted study to estimate the prevalence of GBS colonization in pregnant women in two major university clinics: TSMU The First University and Davit Tvildiani Medical University in 2015-2016 in Tbilisi, Georgia.

\section{Material and Methods:-}

This is a cross-sectional descriptive and analytic study. Anogenital cultures were performed for 1648 pregnant women with gestational age of 35-37 weeks. Two sterile swabs from vagina were obtained by physician and smear test and culture performed in the lab. Direct smear and gram staining to detect bacteria, epithelial cells and the number of white blood cells (WBCs) was performed using the the first swab. The second swab was cultured for GBS on selective chromogenic agar (StrepBSelect Agar,BIO-Rad Laboratories). After 18-24 hour incubation at $37^{\circ} \mathrm{C}$ appearance of blue colons are presumptive identification of GBS. Agglutination test of group-specific polyoside antigens was used for conformation (red agglutination on a green background). Sensitivity of this method is $99,9 \%$.

The results were analyzed via SPSS by Chi square. Significance level was set at 0.05 . The study was approved by The Review Board of Medical University and Prenatal Department and all participants gave written informed consent.

\section{Results:-}

Among 1648 pregnant women (aged 18-42 years) 362 had positive recto- vaginal GBS (21,9\%). No significant difference was found in positive cultures with mother's age, educational level, and history of pregnancy.

\section{Discussion:-}

The overall prevalence for GBS colonization varies different countries is reported 5-40\% depending on the different regions of the world [11] Our data is close to the studies: Grimwood et al. [12] from New Zealand have reported $22 \%$, while Joachim et al. have reported the prevalence of $23 \%$ for GBS colonization. [13] Colonization rate reported was 27.6\% in Portugal [14] 4.7\% in India [15] and 20\% in Taiwan. [16] 
Our results showed that the rate of vaginal colonization in Georgia is approximately the same as the other countries. However, more studies are required to determine the specific rate of vaginal colonization and factors that are responsible for region differences.

\section{Conclusion:-}

The maternal colonization with GBS is a common problem in Georgia. We recommend screening for GBS in all pregnant women and a close observation for all their newborns to be included in national protocol.

\section{Acknowledgments:-}

There is no conflict of interest in this study.

\section{References:-}

1. Edward MS, Baker CJ. Streptococcus agalactia (Group B Streptococcus) In: Mandell GL, Bennett JE, Dolin R, editors. Principles and Practice of Infection Diseases. 7th ed. Elsevier: Churchill Livingstone; 2010.

2. Larsen JW, Sever JL. Group B Streptococcus and pregnancy. Am J Obstet Gynecol. 2010;198(4):440-1.

3. Pettersson K. Perinatal infection with Group B Streptocooci. Semin Fetal Neonate M. 2010;12:193-1974.

4. CDC. Prevention of perinatal group B Streptococcal disease: a public health perspective. Centers for Disease Control and Prevention. MMWR Recomm Rep. 1996;45(7):1-24.

5. Cunningham FG, Williams JW. Williams Obstetrics. 22nd ed. New York, McGraw-Hill: Medical Pub Division; 2005. pp. 39-90.

6. James DK, Steer PH, Weiner CP, Gonik B. High Risk Pregnancy Management option. 3rd ed. Philadelphia: Elsevier Saunders; 2005. pp. 674-90.

7. Behrman RE, Kliegman RM. Nelson Textbook of Pediatrics. 17th ed. Philadelphia: Elsevier Saunders; 2004. pp. 879-83.

8. Stoll BJ, Schuchat A. Maternal carriage of group B Streptococci in developing countries. Pediatr Infect Dis J. 1998;17(6):499-503.

9. Rabiee S, Arab M, Mashouf R. Epidemiologic pattern of vaginal colonization by group B Streptococcus in pregnant women in Hamadan, Central west of Iran. Iran J Med Sci. 2006;31(2):106-68.

10. Aali BS, Abdollahi H, Nakhaee N, Davazdahemami Z, Mehdizadeh A. The association of preterm labor with vaginal colonization of group B streptococci. IJRM. 2007;5(4):191-4.

11. Vaciloto E, Richtmann R, de Paula Fiod Costa H, Kusano EJ, de Almeida MF, Amaro ER. A survey of the incidence of neonatal sepsis by group B Streptococcus during a decade in a Braizilian maternity hospital. Braz J Infect Dis. 2002;6(2):55-62.

12. Joachim A, Mattee ML, Massawe FA, Lyamuya EF. Maternal and neonatal colonization of group B streptococcus at Muhimbili National Hospital in Dar es Salaam, Tanzania: prevalence, risk factors and antimicrobial resistance. BMC public Health. 2009;9:437-437

13. Barcaite E, Bartusevicius A, Tameliene R, Kliucinskas M, Maleckiene L, Nadisauskiene R. Prevalence of maternal group B streptococcus colonization in European countries. Acta Obstet Gynecol Scand. 2008;87(3):260-271.

14. Nomura ML, Passini Jr, Olivier UM, Colil R. Group B streptococcus maternal and neonatal colonization in preterm rupture of membranes and preterm labor. Rev Bras Ginecol obstet. 2009;31(8):397-403

15. Dechen TC, Sumit K, Ranabir P. Correlates of vaginal colonization with group B streptococci among pregnant women. J Glob Infect Vis. 2010;2(3):236-241.

16. Siegel J. Prophylaxis for neonatal group B streptococcus infections. Semin Perinatol. 1998;22(1):33-49. 